\title{
Performance of the Sysmex UF-1000i System in Screening for Significant Bacteriuria in Patients with Bladder Cancer Who Received Bacillus Calmette-Guérin Treatment
}

\author{
Tae Hee Kim, Seung II Jung, Myung Soo Kim, Hyun-Jung Choi ${ }^{1}$, Ho Seok Chung, Eu Chang Hwang, Dong Deuk Kwon \\ Department of Urology, Chonnam National University Medical School, Gwangju, 'Department of Laboratory Medicine, Chonnam National \\ University Hwasun Hospital, Hwasun, Korea
}

Purpose: Diagnosing urinary tract infections by urine culture is time-consuming during a Bacillus Calmette-Guérin treatment. Because the urine culture results take time to collect, patients are treated presumptively, or Bacillus Calmette-Guérin is discontinued before the results. This study evaluated the ability of the Sysmex UF-1000i system to examine the urine bacterial count and determine if it could be used to predict a positive culture.

Materials and Methods: 180 patients who underwent transurethral resection for bladder tumors and received intravesical Bacillus Calmette-Guérin treatment between January 2017 and July 2018 were evaluated prospectively. For patients who received an intravesical Bacillus Calmette-Guérin treatment, urine flow cytometry, and urine cultures were assessed weekly during the treatment period. Results: During Bacillus Calmette-Guérin instillation, 44, 146, and 27 patients had bacteriuria, pyuria, and positive urine nitrite, respectively. Multivariate analysis indicated that the predictors associated with bacteriuria included the urine flow cytometry results of $>100$ bacteria/ $\mu$ (odds ratio, $22.73 ; p<0.001$ ) and positive urine nitrite (odds ratio, 5.67; $p=0.012$ ) at the time of sampling for positive urine culture. The area under the receiver operative characteristic curve for diagnosing bacteriuria by urine flow cytometry was 0.825 . A urine flow cytometry cutoff value of $>100$ bacteria/ $\mu$ l resulted in a sensitivity and specificity of $75 \%$ and $90.91 \%$, respectively. Using the cutoff of $>1,000$ bacteria/ $\mu$ l, the sensitivity and specificity were $50 \%$ and $94.85 \%$, respectively.

Conclusions: Rapid urinary tract infection diagnosis using Sysmex UF-1000i can be used to determine whether to treat an infection and to avoid unnecessary Bacillus Calmette-Guérin discontinuation and urine culture tests.

Keywords: Bacteriuria; Bacillus Calmette-Guérin; Bladder tumor; Flow cytometry; Urinary tract infections

Copyright (c) 2020, Korean Association of Urogenital Tract Infection and Inflammation. All rights reserved. (c) (1) (S) This is an open access article distributed under the terms of the Creative Commons Attribution (c) (1) () Non-Commercial License (http://creativecommons.org/licenses/by-nc/4.0) which permits unrestricted non-commercial use, distribution, and reproduction in any medium, provided the original work is properly cited.
Received: 10 July, 2020

Revised: 13 August, 2020

Accepted: 17 August, 2020
Correspondence to: Seung Il Jung

(iD) https://orcid.org/0000-0003-4864-8175

Department of Urology, Chonnam National University

Hwasun Hospital, 322 Seoyang-ro, Hwasun-eup, Hwasun 58128, Korea

Tel: +82-61-379-7749, Fax: +82-61-379-7750

E-mail: drjsi@yahoo.co.kr
INTRODUCTION

Urinary bladder cancer is one of the most common urological malignancies [1]. Transurethral resection of bladder tumor (TURBT) constitutes the first-line treatment and the diagnostic modality for bladder cancer [2]. 
Intravesical chemotherapy or immunotherapy is used routinely worldwide as an adjunct treatment after TURBT to prevent or delay the recurrence of bladder cancer [3]. On the other hand, irritative symptoms are commonly found in patients who undergo TURBT with or without subsequent intravesical therapy [4]. These symptoms resemble those of an overactive bladder, such as urgency, frequency, and urge incontinence or cystitis [5].

A urinary tract infection (UTI) is an essential determinant in the discontinuation of a Bacillus Calmette-Guérin (BCG) treatment. A urine culture is necessary to confirm a UTI diagnosis and identify the causative agent. Because the urine culture results take one-two days, patients are treated presumptively, or BCG may be discontinued before the culture results are obtained.

Azuma et al. [6] reported that $24 \%$ of patients with non-muscle invasive bladder cancer (NMIBC) have pyuria. On the other hand, for bladder cancer patients receiving BCG treatment, pyuria might also occur as an inflammatory bladder response to BCG. Many patients experience dysuria and lower urinary tract symptoms (LUTS), such as cystitis. Therefore, clinicians often cannot differentiate between pyuria caused by UTI and that caused by an inflammatory response to BCG treatment. In addition, clinicians may treat patients presumptively with antibiotics or discontinue BCG treatment based on pyuria.

Flow cytometry analysis is capable of identifying bacteria, and the Sysmex UF-1000i (Sysmex, Kobe, Japan) flow cytometer has been developed to standardize urine analyses [7]. This system can analyze urine particles rapidly, such as white blood cells (WBC), red blood cells (RBC), bacteria, epithelial cells, and casts effectively and reliably. The unnecessary use of antibiotics can be avoided because these results can be obtained faster than the results of urine cultures. Previous studies [8-14] have shown reliable and valid results using the Sysmex UF-1000i system, which is comparable to the urine culture and microscopic counting results [15].

Thus far, there are no reports on the incidence of pyuria or bacteriuria after TURBT and BCG treatment. Therefore, this study examined the incidence of pyuria or bacteriuria after TURBT and BCG treatment and evaluated the accuracy of the Sysmex UF-1000i system in counting bacteria and WBCs in the urine. The main goal was to determine if it could predict a positive urine culture, allowing the avoidance of unnecessary BCG discontinuation and urine cultures.

\section{MATERIALS AND METHODS}

\section{Ethics Statement}

The Institutional Review Board of the Chonnam National University Hwasun Hospital approved this study (IRB no. CNUHH-2018-007). Ongoing studies on patients with bladder cancer receiving intravesical BCG were approved by the institutional review board, which allowed the collection of retrospective and prospective data on patients treated with BCG. All study procedures were performed according to the Declaration of Helsinki guidelines.

\section{Patients and Samples}

One hundred eighty patients who underwent TURBT and received intravesical BCG treatment between January 2017 and July 2018 were evaluated prospectively. Urinalyses and urine cultures were performed at the baseline and one-two weeks after TURBT. In patients who underwent an intravesical BCG treatment, urinalyses and urine cultures were performed weekly during the BCG treatment period. Patients diagnosed with muscle invasive urothelial carcinoma after TURBT and those showing pathological stage T3 or T4 bladder cancer were excluded.

Intravesical BCG therapy was determined after surgery, and a symptomatic questionnaire, urine analysis, and urine culture were performed. The treatment is stopped for one week if a patient complains of symptoms of fever or severe irritation. The discontinuation of this study was $30 \%$. Bacteriuria was predicted based on the subjective symptoms, patient body temperature, pyuria, and urine nitrite. Physicians should use discretion in the management of symptoms during BCG therapy and the prophylactic use of antibiotics.

The accuracy of the Sysmex UF-1000i in counting bacteria in the urine was evaluated to determine if it could be used to predict a positive culture. Samples were excluded if urine analysis and urine culture were not performed within 24 hours of each other. The midstream urine specimens were collected in sterile containers for urine analyses and cultures. The urine specimens were placed in 10-ml urine sediment centrifuge tubes and sent for chemical analysis and a laser flow cytometry (Sysmex UF-1000i) examination in the laboratory. The Sysmex UF-1000i analysis was performed 
within 30 minutes of collection.

\section{Sysmex UF-1000i}

In the authors' hospital, the standard urinalysis is performed by the Sysmex UF-1000i and one chemistry analyzer. The results of sediment analysis are presented as the number per $\mu l$ and number per high power field (HPF).

The Sysmex UF-1000i is a fully automated fluorescence flow cytometer that uses a $635-\mathrm{nm}$ semiconductor diode laser to separate the bacteria and sediment into two analytical channels without the need to centrifuge the urine specimens. In the bacterial channel, the urine specimen is mixed at $42{ }^{\circ} \mathrm{C}$. A special diluent, which increases the bacterial cell wall permeability, allows the polymethine fluorescent dye to stain the bacterial DNA. Each bacterial particle, exposed to a 635-nm laser beam, emits various degrees of forward-scattered light and side-scattered fluorescent light and are then counted to generate the $\mathrm{BACT} / \mathrm{ml}$. The sediment channel analyzes the WBCs, RBCs, yeast-like cells, epithelial cells, crystals, casts, spermatozoa, and mucus. The particles/ml can then be converted to the traditional number/HPF by multiplying specific factors. Pyuria was defined as WBC >10/HPF.

\section{Statistical Analysis}

The predictive validities of nitrite, pyuria (>10/HPF), and $\mathrm{BACT} / \mathrm{ml}$ for determining significant bacteriuria were analyzed by logistic regression. Receiver operating characteristic (ROC) curve analysis was used to determine the best cutoff point to represent significant bacteriuria. A p-value $<0.05$ was considered significant.

\section{RESULTS}

\section{Baseline Characteristics of the Patients and Screening for Significant Bacteriuria}

Table 1 lists the baseline characteristics of patients who underwent TURBT. Bacteriuria is defined as the presence of bacteria in a urine culture during BCG treatment. Only bacteriuria after the BCG therapy was included in the bacteriuria group.

Table 2 lists the urine culture, microscopic analysis, and urine flow cytometry results. Of the 180 patients evaluated, 155 (86.1\%) were male, and the median age was 70 (interquartile range 62-77) years. During BCG instillation, 44 (24.4\%), 146 (81.1\%), and $27(15.0 \%)$ patients had

Table 1. Baseline characteristics of patients who underwent transurethral resection of bladder tumor

\begin{tabular}{|c|c|c|c|c|}
\hline Variable & Total $(n=180)$ & $\begin{array}{l}\text { BCG treatmentwith } \\
\text { bacteriuria }^{\text {a) }}(n=44)\end{array}$ & $\begin{array}{l}\text { BCG treatment without } \\
\text { bacteriuria }^{a)}(n=136)\end{array}$ & p-value \\
\hline Median age $(y)$ & $70.0(62.0-77.0)$ & $76.0(70.0-79.5)$ & $69.0(60.0-77.0)$ & 0.002 \\
\hline Male & $155(86.1)$ & $27(61.4)$ & $128(94.1)$ & $<0.001$ \\
\hline Smoking & & & & 0.004 \\
\hline Non-smoker & $93(51.7)$ & $31(70.5)$ & $62(45.6)$ & \\
\hline Current and ex-smoker & $87(48.3)$ & $13(29.5)$ & $74(54.4)$ & \\
\hline BMI & $24.6(22.6-26.7)$ & $23.9(21.9-25.9)$ & $24.7(22.8-26.9)$ & 0.355 \\
\hline Hypertension & $87(48.3)$ & $23(52.3)$ & $64(47.1)$ & 0.521 \\
\hline History of nephroureterectomy & $21(11.7)$ & $9(20.5)$ & $12(8.8)$ & 0.035 \\
\hline Tumor stage & & & & 0.365 \\
\hline Tis & $10(5.6)$ & $3(6.8)$ & $7(5.1)$ & \\
\hline Ta & $111(61.7)$ & $29(65.9)$ & $82(60.3)$ & \\
\hline T1 & $59(32.8)$ & $12(27.3)$ & $47(34.6)$ & \\
\hline Grade & & & & 0.896 \\
\hline PUNLMP & $2(1.1)$ & $1(2.3)$ & $1(0.7)$ & \\
\hline Low & $48(26.7)$ & $11(25.0)$ & $36(26.5)$ & \\
\hline High & $131(72.8)$ & $32(72.7)$ & $99(72.8)$ & \\
\hline $\begin{array}{l}\text { Immediate intravesical treatment } \\
\text { BCG treatment }\end{array}$ & $63(35.0)$ & $14(31.8)$ & $49(36.0)$ & 0.572 \\
\hline Induction only & $148(82.2)$ & $35(79.5)$ & $113(83.1)$ & 0.579 \\
\hline Induction and maintenance & $32(17.8)$ & $9(20.5)$ & 23 (16.9) & 0.579 \\
\hline Antibiotics during BCG instillation & & & & 0.110 \\
\hline Prophylactic use & $68(37.8)$ & $12(27.3)$ & $56(41.2)$ & \\
\hline Therapeutic use & $7(3.9)$ & $6(13.6)$ & $1(0.7)$ & \\
\hline Discontinuation of BCG instillation & $44(24.4)$ & $17(38.6)$ & $27(19.9)$ & 0.012 \\
\hline
\end{tabular}

Values are presented as median (interquartile range) or number (\%).

BCG: Bacillus Calmette-Guérin, BMI: body mass index, PUNLMP: papillary urothelial neoplasm of low malignant potential.

${ }^{\text {a) }}$ The definition of bacteriuria is the presence of bacteria in the urine culture during BCG treatment. 
Table 2. Urine culture, microscopic analysis, and urine flow cytometry results

\begin{tabular}{|c|c|c|c|c|}
\hline Variable & Total $(n=180)$ & $\begin{array}{c}\text { BCG treatmentwith } \\
\text { bacteriuria } \\
(\mathrm{n}=44)\end{array}$ & $\begin{array}{l}\text { BCG treatment } \\
\text { without bacteriuria } \\
(n=136)\end{array}$ & p-value \\
\hline Preoperative bacteriuria & $10(5.6)$ & $5(11.4)$ & $5(3.7)$ & 0.051 \\
\hline Preoperative pyuria & $59(32.8)$ & $14(31.8)$ & $45(33.1)$ & 0.582 \\
\hline Preoperative positive urine nitrite & $7(3.9)$ & $1(2.3)$ & $6(4.4)$ & 0.055 \\
\hline Preoperativeurine flow cytometry bacterial count $(/ \mu \mathrm{l})$ & & & & 0.451 \\
\hline $0-100$ & $137(76.1)$ & $27(61.4)$ & $110(80.9)$ & \\
\hline$>100$ & $31(17.2)$ & $13(29.5)$ & $18(13.2)$ & \\
\hline$>1,000$ & $11(6.1)$ & $3(6.8)$ & $8(5.9)$ & \\
\hline$>10,000$ & $1(0.6)$ & $1(2.3)$ & 0 & \\
\hline Immediate postoperative bacteriuria & $12(6.7)$ & $6(13.6)$ & $6(4.4)$ & 0.030 \\
\hline Immediate postoperative pyuria & $134(74.4)$ & $36(81.8)$ & $98(72.1)$ & 0.176 \\
\hline Immediate postoperative positive urine nitrite & $15(8.3)$ & $7(15.9)$ & $8(5.9)$ & 0.035 \\
\hline Immediate postoperative urine flow cytometry bacterial count $(/ \mu \mathrm{l})$ & & & & 0.007 \\
\hline $0-100$ & $138(76.7)$ & $28(63.6)$ & $110(80.9)$ & \\
\hline$>100$ & $32(17.8)$ & $10(22.7)$ & $22(16.2)$ & \\
\hline$>1,000$ & $6(3.3)$ & $2(4.5)$ & $4(2.9)$ & \\
\hline$>10,000$ & $4(2.3)$ & $4(9.1)$ & 0 & \\
\hline Bacteriuria after BCG treatment & $44(24.4)$ & $44(100.0)$ & 0 & \\
\hline Pyuria after BCG treatment & $146(81.1)$ & $41(93.2)$ & $105(77.2)$ & 0.027 \\
\hline Positive urine nitrite after BCG treatment & $27(15.0)$ & $20(45.5)$ & $7(5.1)$ & $<0.001$ \\
\hline Urine flow cytometry bacterial count $(/ \mu \mathrm{l})$ after BCG treatment & & & & $<0.001$ \\
\hline $0-100$ & $106(58.9)$ & $4(9.1)$ & $102(75.0)$ & \\
\hline$>100$ & $45(25.0)$ & $18(40.9)$ & 27 (19.9) & \\
\hline$>1,000$ & $20(11.1)$ & $14(31.8)$ & $6(4.4)$ & \\
\hline$>10,000$ & $9(5.0)$ & $8(18.2)$ & $1(0.7)$ & \\
\hline Bacteriuria 1 year after BCG treatment & $22(12.2)$ & $8(38.1)$ & $14(10.3)$ & 0.083 \\
\hline Pyuria 1 year after BCG treatment & $21(11.7)$ & $8(36.4)$ & $13(9.6)$ & 0.141 \\
\hline Positive urine nitrite one year after BCG treatment & $8(4.4)$ & $5(22.7)$ & $3(2.2)$ & 0.025 \\
\hline Urine flow cytometry bacterial count $(/ \mu \mathrm{l}) 1$ year after BCG treatment & & & & 0.001 \\
\hline $0-100$ & $66(36.7)$ & $13(59.1)$ & $53(39.0)$ & \\
\hline$>100$ & $11(6.1)$ & 3 (13.6) & $8(5.9)$ & \\
\hline$>1,000$ & $4(2.2)$ & $3(13.6)$ & $1(0.7)$ & \\
\hline$>10,000$ & $4(2.2)$ & $3(13.6)$ & $1(0.7)$ & \\
\hline
\end{tabular}

Values are presented as number (\%).

BCG: Bacillus Calmette-Guérin.

bacteriuria, pyuria, and positive urine nitrite, respectively. Multivariate analysis indicated that the predictors associated with bacteriuria included the urine flow cytometry results of $>100$ bacteria/ $\mu$ l (OR, 22.73; p <0.001) and positive urine nitrite (OR, 5.67; $\mathrm{p}=0.012)$ at that time of sampling for a positive urine culture.

\section{ROC Curve Analysis}

The ROC curves were used to define the optimal cutoff point of $\mathrm{BACT} / \mathrm{ml}$ for predicting a positive culture (single bacterial growth $\geq 10^{5} \mathrm{CFU} / \mathrm{ml}$ ). The area under the ROC curve for the diagnosis of bacteriuria by urine flow cytometry was 0.872 (Fig. 1). A flow cytometry cut off value of $>100$ bacteria/ $\mu 1$ resulted in a sensitivity and specificity of $75 \%$ and $90.91 \%$, respectively. Using the cutoff value of $>1,000$ bacteria/ $\mu$, the sensitivity and specificity were $50 \%$ and 94.85\%, respectively (Table 3). Pyuria showed low specificity (28.08\%), and positive urine nitrite showed low sensitivity

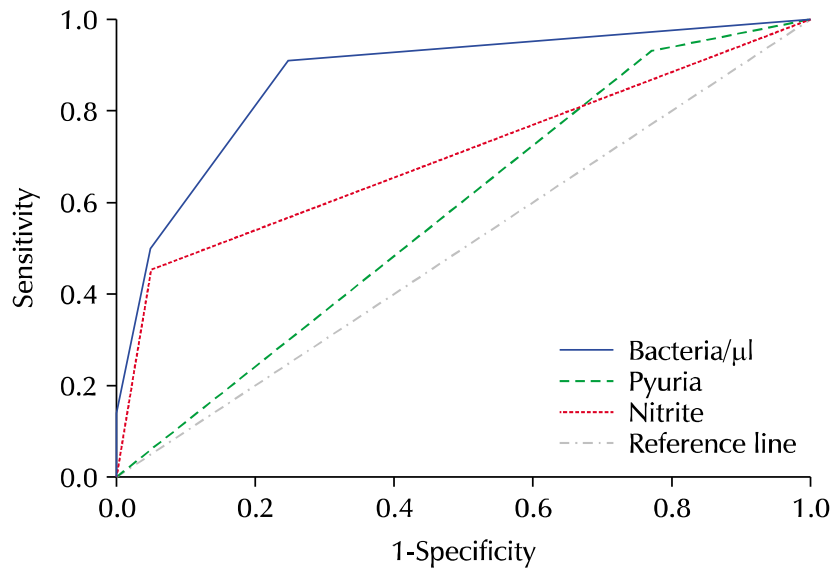

Fig. 1. Receiver operating characteristic (ROC) curves for bacteriuria detection using urine flow cytometry (when a bacterial count $<10^{5}$ $\mathrm{CFU} / \mathrm{ml}$ is used as the gold standard definition for negative cultures). The area under the ROC curve for the diagnosis of bacteriuria by urine flow cytometry was 0.872 . 
Table 3. Predictive value of urine microscopy and urine flow cytometry for bacteriuria diagnosis

\begin{tabular}{|c|c|c|c|c|}
\hline & Specificity (\%) & Sensitivity (\%) & PPV (\%) & NPV (\%) \\
\hline Pyuria-positive & 28.08 & 91.18 & 22.79 & 93.18 \\
\hline Nitrite-positive & 94.85 & 54.55 & 77.42 & 86.58 \\
\hline Urine flow cytometry bacterial count $>100 / \mu \mathrm{l}$ & 90.91 & 75.00 & 54.05 & 96.23 \\
\hline Urine flow cytometry bacterial count $>1,000 / \mu \mathrm{l}$ & 94.85 & 50.00 & 75.86 & 85.43 \\
\hline
\end{tabular}

PPV: positive predictive value, NPV: negative predictive value.

Table 4. Bacteriuria analysis by each diagnostic method according to cultured bacteria

\begin{tabular}{|c|c|c|c|c|c|c|c|c|}
\hline \multirow{2}{*}{ Microorganism } & \multirow{2}{*}{$\mathrm{N}$} & \multirow{2}{*}{$\begin{array}{c}\begin{array}{c}\text { Urine } \\
\text { nitrite test }\end{array} \\
(+)\end{array}$} & \multirow{2}{*}{$\begin{array}{c}\text { Microscopic } \\
\text { urine analysis } \\
\text { Pyuria } \\
(\text { WBC }>10)\end{array}$} & \multicolumn{5}{|c|}{ UF bacterial count } \\
\hline & & & & 0 & 1 & 2 & 3 & 4 \\
\hline Escherichia coli & 13 & 9 & 13 & 1 & 5 & 4 & 2 & 1 \\
\hline Enterococcus faecalis & 8 & & 7 & & 3 & 4 & 1 & \\
\hline Staphylococcus epidermidis & 4 & 2 & 4 & & 3 & 1 & & \\
\hline Coagulase negative Staphylococcus & 3 & 2 & 3 & 1 & 1 & 1 & & \\
\hline Streptococcus agalactiae Gr. B & 3 & & 3 & & 1 & 2 & & \\
\hline Klebsiella pneumoniae & 2 & 2 & 2 & & 1 & & & 1 \\
\hline Non-hemolytic Streptococcus & 2 & 1 & 2 & 1 & 1 & & & \\
\hline Proteus mirabilis & 2 & 2 & 2 & & & & 2 & \\
\hline Streptococcus mitis/oralis & 2 & & 1 & 1 & 1 & & & \\
\hline Aeromonas hydrophila/caviae & 1 & & & & & & & 1 \\
\hline Corynebacterium striatum & 1 & 1 & 1 & & & 1 & & \\
\hline Enterococcus faecium & 1 & & 1 & & 1 & & & \\
\hline Morganella morganii & 1 & 1 & 1 & & & 1 & & \\
\hline Viridans Streptococcus & 1 & & 1 & & 1 & & & \\
\hline Total & 44 & 20 & 41 & 4 & 18 & 14 & 5 & 3 \\
\hline
\end{tabular}

WBC: white blood cell, UF: urine flow cytometer.

(54.55\%).

\section{Differences between the Bacterial Species}

In the case of Escherichia coli, nitrite positive, pyuria, and urine flow cytometer (UF) bacterial counts $>100$ were 9/13 (69.2\%), 13/13 (100\%), and 12/13 (92.3\%), respectively (Table 4). When the UF count was 0 to 1,000 , it tended to be nitrite (-) (Fig. 2A). In the case of finding pyuria, the UF count was evenly distributed, reflecting the high sensitivity of microscopic pyuria (Fig. 2B).

\section{DISCUSSION}

Because UTIs are common in bladder cancer patients undergoing a BCG treatment, reliable automatic screening systems are needed to reduce unnecessary BCG discontinuation. This study evaluated the ability of the Sysmex UF-1000i system to predict a positive culture accurately so that unnecessary BCG discontinuation and urine cultures can be avoided.
The predictors associated with bacteriuria in patients undergoing a BCG treatment included urine flow cytometry results of $>100$ bacteria/ $\mu$ l $(O R, 22.73 ; p<0.001)$ and positive urine nitrite $(O R, 5.67 ; p=0.012)$ at the time of sampling for a positive urine culture. A urine flow cytometry cutoff value of $>100$ bacteria/ $\mu$ l resulted in a sensitivity and specificity of $75 \%$ and $90.91 \%$, respectively. Using the cutoff $>1,000$ bacteria/ $\mu$ l, the sensitivity and specificity were $50 \%$ and $94.85 \%$, respectively. On the other hand, the urine nitrite positivity had low sensitivity (54.55\%) for bacteriuria after BCG treatment was initiated.

In this study, pyuria showed a low specificity for bacteriuria after the BCG treatment. Azuma et al. [ $[$ ] reported that $24 \%$ of patients with non-muscle-invasive urothelial carcinoma demonstrated pyuria, as defined by WBCs $\geq$ 10/HPF in their urine. Satake et al. [16] reported that 116 out of 237 (48.9\%) of patients with NMIBC had preoperative pyuria. On the other hand, unlike the present study, they only assessed pyuria. The rate of bacteriuria was not determined. In this study, the rates of preoperative pyuria 
A

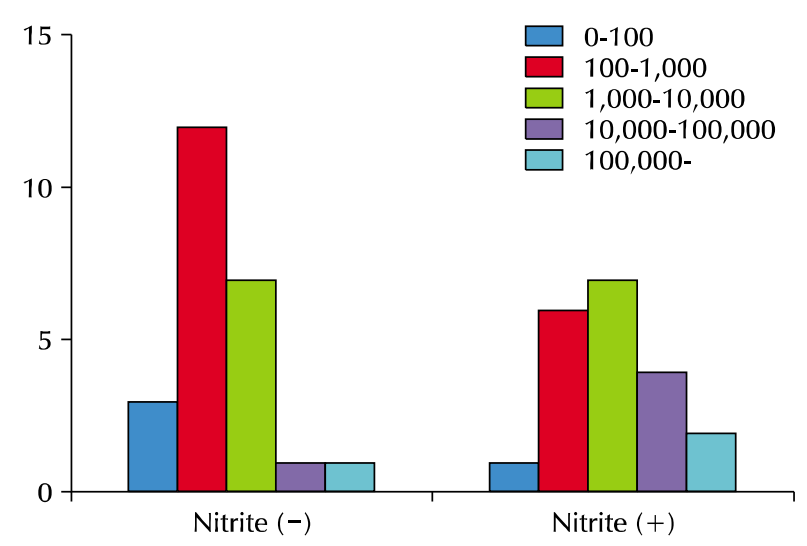

B

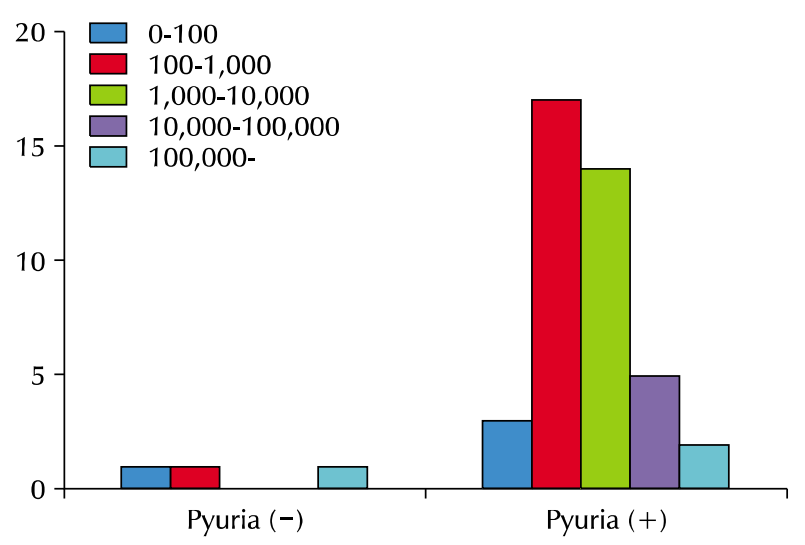

Fig. 2. (A) Urine flow cytometer (UF) bacterial counts by nitrite test. (B) UF bacterial counts by pyuria.

and bacteriuria were $32.8 \%$ and $5.6 \%$, respectively. Pyuria in bladder cancer patients undergoing TURBT and receiving BCG treatment increased up to $74-81 \%$ due to postoperative inflammation or an inflammatory response to BCG treatment. Despite the high rate of pyuria after the TURBT and BCG treatment, the rate of postoperative bacteriuria was $6.8 \%$. The rate of bacteriuria after the BCG treatment increased to $24.3 \%$. Therefore, pyuria cannot predict bacteriuria because of its low specificity and low positive predictive value for bacteriuria.

The incidence of postoperative UTIs in TURBT ranged from $10 \%$ to $40 \%[17,18]$. Therefore, prophylactic antibiotics are recommended. In this study, all patients received prophylactic antibiotics for TURBT, and the postoperative UTI rate did not increase. The bacteriuria rate increased after the BCG treatment because antibiotics are not administered routinely after intravesical BCG instillation. Before BCG treatment, the bacteriuria rate ranged from $18.1 \%$ to $25 \%$ [19,20]. In this study, $37.8 \%$ of patients received prophylactic antibiotics after BCG instillation based on clinical symptoms and pyuria to reduce BCG complications. On the other hand, the bacteriuria rate after BCG treatment increased to $24.3 \%$.

Adjuvant intravesical immunotherapy with BCG is the standard treatment after surgery in patients with high-risk NMIBC [21], but BCG therapy is associated with a significant risk of local and systemic adverse events [22]. Because UTIs are regarded as a risk factor for BCG complications, symptomatic UTIs are an absolute contraindication to the therapy [21]. Some experts believe that bacterial cystitis causes trauma to the BCG barrier, allowing it to reach the bloodstream [23]. On the other hand, an antimicrobial treatment delays adjuvant oncological treatment and potentially contributes to the increase in bacterial resistance to antibiotics, with no apparent clinical benefit.

The predisposing factors for intravesical BCG related complications are not well known. Some conditions may result in hematogenous dissemination of the bacilli (i.e., disruption of the urothelial barrier due to traumatic urinary catheterization, early instillation after TURBT, or concurrent UTI) [24].

Urologists are reluctant to administer BCG to patients with infections because of the possible risk of bacterial- or BCG-related sepsis or an abrogated response to BCG [25]. Poletajew et al. [26] reported in their systematic review that asymptomatic bacteriuria does not adversely affect the safety and efficacy of intravesical BCG immunotherapy. The authors suggested that there was no evidence to support the routine screening for and treatment of asymptomatic bacteriuria in bladder cancer patients scheduled for intravesical BCG instillations. Therefore, antibiotics are not administered routinely after intravesical BCG instillation [26]. Many patients receiving BCG treatment have LUTS, such as dysuria, frequency, urgency, and hematuria, which cannot be differentiated from UTI symptoms. Because urine culture results take one-two days, patients are treated presumptively based on pyuria, and LUTS or BCG treatment is discontinued before obtaining the urine culture results. Although symptomatic UTIs remains an absolute contraindication for BCG therapy, bacteriuria cannot be assessed on the day of the BCG treatment. Therefore, evidence of pyuria on urine analysis is normally used to determine the presence of a 
UTI. On the other hand, in this study, pyuria was found to have low specificity for bacteriuria and was observed frequently after the BCG treatment. As a result, patients should not be treated presumptively based on pyuria or be discontinued from receiving BCG treatment based on this finding.

In this study, positive urine nitrite (OR, 5.67; p=0.012) at the time of sampling for a positive urine culture was a risk factor for bacteriuria. On the other hand, urine nitrite positivity had low sensitivity for bacteriuria after the BCG treatment was initiated. A nitrite test is a standard component of a urinary test strip. A positive test result for nitrites in the urine is called nitrituria. This test is commonly used to diagnose UTIs. A positive nitrite test result suggests that the cause of the UTI is a gram-negative organism, the most common being $E$. coli. The reason for the presence of nitrites in a UTI is the bacterial conversion of endogenous nitrates to nitrites. This may be a sign of infection. Other parameters, such as the leukocyte esterase levels and urine WBC count, and symptoms, such as dysuria, urinary urgency, fevers, and chills, must be correlated to diagnose an infection.

False-negative nitrite test results in UTIs occur in cases involving a low CFU count or in recently voided or dilute urine [27]. In addition, a nitrite test does not detect organisms unable to reduce nitrate to nitrite, such as enterococci, staphylococci (Staphylococcus saprophyticus), acinetobacter, or adenovirus [27].

According to this study, a urine flow cytometry result of $>100$ bacteria/ $\mu$ l (OR, 22.73; p<0.001) at the time of sampling for a positive urine culture was a predictor for bacteriuria; these results could be determined before the urine cultures. The area under the ROC curve for the diagnosis of bacteriuria by urine flow cytometry was 0.825 . A flow cytometry cutoff value of $>100$ bacteria/ $\mu$ l resulted in a sensitivity and specificity of $75 \%$ and $90.91 \%$, respectively. Using the cutoff of $>1,000$ bacteria/ $\mu$, the sensitivity and specificity were $50 \%$ and $94.85 \%$, respectively. There are many opposing opinions regarding the optimal cutoff point that can be used with the Sysmex UF-1000i system. According to some studies, the cutoff point ranges from 25 to 230 bacteria/ $\mu 1$ [9,10,14,28-30]. In the present study, an optimal flow cytometry cutoff value of $>100$ bacteria/ $\mu$ l was established.

This study showed that flow cytometry using the Sysmex UF-1000i system is a useful method to exclude a UTI and may contribute to the reduction of unnecessary BCG discontinuation and urine cultures. The cutoff points used with the Sysmex UF-1000i in this study resulted in a decrease in the number of urine cultures drawn.

The limitation of this study is that there is no consensus on the optimal management of symptoms and the use of antibiotics in patients who received BCG therapy. The purpose of this study was to show the validation of Sysmex UF-1000i for bacteriuria in patients who received BCG therapy. Therefore, physicians should use discretion when managing the symptoms during BCG therapy and the use of antibiotics. Additional prospective studies will be needed to evaluate the management further according to the results of flow cytometry. A prospective study will be needed to follow the clinical outcomes of specific antibiotic use based on the timely result provided by Sysmex UF-1000i.

\section{CONCLUSIONS}

The rate of pyuria and bacteriuria increased after the BCG treatment. Because UTIs are considered a risk factor for BCG complications, symptomatic UTIs are an absolute contraindication to the therapy. On the other hand, many patients receiving BCG have LUTS, such as dysuria, frequency, and urgency caused by irritation associated with the BCG treatment, which cannot be differentiated from the UTI symptoms. In this situation, the rapid diagnosis of UTI using Sysmex UF-1000i (results of $>100$ bacteria/ $\mu$ ) and positive urine nitrite may help determine whether to treat an infection and avoid unnecessary BCG discontinuation and urine cultures.

\section{CONFLICT OF INTEREST}

No potential conflict of interest relevant to this article was reported.

\section{ACKNOWLEDGMENTS}

This study was supported by a grant (HCRI 19017) from the Chonnam National University Hwasun Hospital Institute for Biomedical Science. 


\section{AUTHOR CONTRIBUTIONS}

T.H.K. participated in data collection and wrote the manuscript. H.J.C. participated in data collection. M.S.K., H.S.C., and E.C.H. participated in the study design and performed the statistical analysis. S.I.J. and D.D.K. participated in the study design and coordination and helped to draft the manuscript. All authors read and approved the final manuscript.

\section{ORCID}

Tae Hee Kim, https://orcid.org/0000-0002-0546-0431 Seung Il Jung, https://orcid.org/0000-0003-4864-8175

Myung Soo Kim, https://orcid.org/0000-0003-1456-2154

Hyun-Jung Choi, https://orcid.org/0000-0003-2901-5962

Ho Seok Chung, https://orcid.org/0000-0001-9883-1539

Eu Chang Hwang, https://orcid.org/0000-0002-2031-124X

Dong Deuk Kwon, https://orcid.org/0000-0002-4544-5420

\section{REFERENCES}

1. Haresh KP, Julka PK, Sharma DN, Rath GK, Prabhakar R, Seth A. A prospective study evaluating surgery and chemo radiation in muscle invasive bladder cancer. J Cancer Res Ther 2007;3: 81-5.

2. Divrik RT, Sahin AF, Yildirim U, Altok M, Zorlu F. Impact of routine second transurethral resection on the long-term outcome of patients with newly diagnosed pT1 urothelial carcinoma with respect to recurrence, progression rate, and disease-specific survival: a prospective randomised clinical trial. Eur Urol 2010;58:185-90.

3. Hall MC, Chang SS, Dalbagni G, Pruthi RS, Seigne JD, Skinner $\mathrm{EC}$, et al. Guideline for the management of nonmuscle invasive bladder cancer (stages Ta, T1, and Tis): 2007 update. J Urol 2007;178:2314-30.

4. Thrasher JB, Crawford ED. Complications of intravesical chemotherapy. Urol Clin North Am 1992;19:529-39.

5. Abrams P, Andersson KE, Birder L, Brubaker L, Cardozo L, Chapple $\mathrm{C}$, et al. Fourth International Consultation on Incontinence Recommendations of the International Scientific Committee: evaluation and treatment of urinary incontinence, pelvic organ prolapse, and fecal incontinence. Neurourol Urodyn 2010;29:213-40.

6. Azuma T, Nagase Y, Oshi M. Pyuria predicts poor prognosis in patients with non-muscle-invasive bladder cancer. Clin Genitourin Cancer 2013;11:331-6.

7. Millan-Lou MI, Garcia-Lechuz JM, Ruiz-Andres MA, Lopez C, Aldea MJ, Revillo MJ, et al. Validation and search of the ideal cut-off of the Sysmex UF-1000i® flow cytometer for the diagnosis of urinary tract infection in a tertiary hospital in Spain. Front Med (Lausanne) 2018;5:92.

8. Broeren MA, Bahceci S, Vader HL, Arents NL. Screening for urinary tract infection with the Sysmex UF-1000i urine flow cytometer. J Clin Microbiol 2011;49:1025-9.

9. De Rosa R, Grosso S, Bruschetta G, Avolio M, Stano P, Modolo $M L$, et al. Evaluation of the Sysmex UF1000i flow cytometer for ruling out bacterial urinary tract infection. Clin Chim Acta 2010;411:1137-42.

10. Kadkhoda K, Manickam K, Degagne P, Sokolowski P, Pang P, Kontzie N, et al. UF-1000i flow cytometry is an effective screening method for urine specimens. Diagn Microbiol Infect Dis 2011;69:130-6.

11. Manoni F, Tinello A, Fornasiero L, Hoffer P, Temporin V, Valverde $S$, et al. Urine particle evaluation: a comparison between the UF-1000i and quantitative microscopy. Clin Chem Lab Med 2010;48:1107-11.

12. Shang YJ, Wang QQ, Zhang JR, Xu YL, Zhang WW, Chen Y, et al. Systematic review and meta-analysis of flow cytometry in urinary tract infection screening. Clin Chim Acta 2013;424: 90-5.

13. van der Zwet WC, Hessels J, Canbolat F, Deckers MM. Evaluation of the Sysmex UF-1000i ${ }^{\circledR}$ urine flow cytometer in the diagnostic work-up of suspected urinary tract infection in a Dutch general hospital. Clin Chem Lab Med 2010;48:1765-71.

14. Wang J, Zhang Y, Xu D, Shao W, Lu Y. Evaluation of the Sysmex UF-1000i for the diagnosis of urinary tract infection. Am J Clin Pathol 2010;133:577-82.

15. Yang CC, Yang SS, Hung HC, Chiang IN, Peng CH, Chang SJ. Rapid differentiation of cocci/mixed bacteria from rods in voided urine culture of women with uncomplicated urinary tract infections. J Clin Lab Anal 2017;31:e22071.

16. Satake N, Ohno Y, Nakashima J, Ohori M, Tachibana M. Prognostic value of preoperative pyuria in patients with non-muscle-invasive bladder cancer. Int J Urol 2015;22:645-9.

17. Janknegt RA. Prophylaxis in urological surgery. Infection 1992;20 Suppl 3:S213-6; discussion S217-20, S224.

18. Westenfelder M, Rosset K, Pelz K. Development of nosocomial and iatrogenic urinary tract infections (UTI) following urological interventions. A prospective clinical study. Scand J Urol Nephrol Suppl 1987;104:59-63.

19. Herr HW. Intravesical bacillus Calmette-Guerin outcomes in patients with bladder cancer and asymptomatic bacteriuria. J Urol 2012;187:435-7.

20. Zhao LC, Meeks JJ, Helfand BT, Ross FR, Herr HW, Kundu SD. Screening urine analysis before bacille Calmette-Guerin instillation does not reduce the rate of infectious complications. BJU Int 2012;109:1819-21.

21. Babjuk M, Bohle A, Burger M, Capoun O, Cohen D, Comperat $E M$, et al. EAU guidelines on non-muscle-invasive urothelial carcinoma of the bladder: update 2016. Eur Urol 2017;71: 


\section{7-61.}

22. Brausi M, Oddens J, Sylvester R, Bono A, van de Beek C, van Andel G, et al. Side effects of Bacillus Calmette-Guerin (BCG) in the treatment of intermediate- and high-risk Ta, T1 papillary carcinoma of the bladder: results of the EORTC genito-urinary cancers group randomised phase 3 study comparing one-third dose with full dose and 1 year with 3 years of maintenance BCG. Eur Urol 2014;65:69-76.

23. Decaestecker K, Oosterlinck W. Managing the adverse events of intravesical bacillus Calmette-Guerin therapy. Res Rep Urol 2015;7:157-63.

24. Perez-Jacoiste Asin MA, Fernandez-Ruiz M, Lopez-Medrano F, Lumbreras C, Tejido A, San Juan R, et al. Bacillus Calmette-Guerin (BCG) infection following intravesical BCG administration as adjunctive therapy for bladder cancer: incidence, risk factors, and outcome in a single-institution series and review of the literature. Medicine (Baltimore) 2014;93:236-54.

25. Billips BK, Forrestal SG, Rycyk MT, Johnson JR, Klumpp DJ, Schaeffer AJ. Modulation of host innate immune response in the bladder by uropathogenic Escherichia coli. Infect Immun
2007;75:5353-60.

26. Poletajew S, Zapala P, Radziszewski P. Safety and efficacy of intravesical Bacillus Calmette-Guerin immunotherapy in patients with non-muscle-invasive bladder cancer presenting with asymptomatic bacteriuria: a systematic review. Urol Int 2017;99:1-5.

27. Deville WL, Yzermans JC, van Duijn NP, Bezemer PD, van der Windt DA, Bouter LM. The urine dipstick test useful to rule out infections. A meta-analysis of the accuracy. BMC Urol 2004;4:4.

28. Jolkkonen S, Paattiniemi EL, Karpanoja P, Sarkkinen $H$. Screening of urine samples by flow cytometry reduces the need for culture. J Clin Microbiol 2010;48:3117-21.

29. Manoni F, Fornasiero L, Ercolin M, Tinello A, Ferrian M, Hoffer $P$, et al. Cutoff values for bacteria and leukocytes for urine flow cytometer Sysmex UF-1000i in urinary tract infections. Diagn Microbiol Infect Dis 2009;65:103-7.

30. Pieretti B, Brunati P, Pini B, Colzani C, Congedo P, Rocchi M, et al. Diagnosis of bacteriuria and leukocyturia by automated flow cytometry compared with urine culture. J Clin Microbiol 2010;48:3990-6. 